\title{
Expert versus generalist inserters for peripheral intravenous catheter insertion: a pilot randomised controlled trial
}

Nicole Marsh ${ }^{1,2,3}$, Joan Webster ${ }^{1,2,4}$, Emily Larsen ${ }^{1,2,3}$, Jodie Genzel ${ }^{1,2,3}$, Marie Cooke ${ }^{2,3}$, Gabor Mihala ${ }^{3,5,6}$, Sue Cadigan ${ }^{1}$ and Claire M Rickard ${ }^{1,2,3^{*}}$ (D)

\begin{abstract}
Background: Peripheral intravenous catheters (PVCs) are essential invasive devices, with 2 billion PVCs sold each year. The comparative efficacy of expert versus generalist inserter models for successful PVC insertion and subsequent reliable vascular access is unknown.

Methods: A single-centre, parallel-group, pilot randomised controlled trial (RCT) of 138 medical/surgical patients was conducted in a large tertiary hospital in Australia to compare PVC insertion by (1) a vascular access specialist (VAS) or (2) any nursing or medical clinician (generalist model). The primary outcome was the feasibility of a larger $\mathrm{RCT}$ as established by predetermined criteria (eligibility, recruitment, retention, protocol adherence). Secondary outcomes were PVC failure: phlebitis, infiltration/extravasation, occlusion, accidental removal or partial dislodgement, local infection or catheter-related bloodstream infection; dwell time; insertion success, insertion attempts; patient satisfaction; and procedural cost-effectiveness.
\end{abstract}

Results: Feasibility outcomes were achieved: $92 \%$ of screened patients were eligible; two patients refused participation; there was no attrition or missing outcome data. PVC failure was higher with generalists (27/50, 54\%) than with VASs (33/69, 48\%) (228 versus 217 per 1000 PVC days; incidence rate ratio 1.05, 95\% confidence interval 0.61-1.80). There were no local or PVC-related infections in either group. All PVCs $(n=69)$ were successfully inserted in the VAS group. In the generalist group, 19 (28\%) patients did not have a PVC inserted. There were inadequate data available for the costeffectiveness analysis, but the mean insertion procedure time was 2 min in the VAS group and 11 min in the generalist group. Overall satisfaction with the PVC was measured on an 11-point scale $(0=$ not satisfied and $10=$ satisfied $)$ and was higher in the VAS group $(n=43$; median $=7)$ compared to the generalist group $(n=20$; median $=4.5)$. The multivariable model identified medical diagnosis and bed-bound status as being significantly associated with higher PVC failure, and securement with additional non-sterile tape was significantly associated with lower PVC failure.

Conclusion: This pilot trial confirmed the feasibility and need for a large, multicentre RCT to test these PVC insertion models.

Trial registration: Australian New Zealand Clinical Trials Registry, ACTRN12616001675415. Registered on 6 December 2016.

Keywords: Intravenous, Vascular access devices, Randomised controlled trial, Phlebitis

\footnotetext{
* Correspondence: c.rickard@griffith.edu.au

${ }^{1}$ Royal Brisbane and Women's Hospital, Herston, QLD, Australia

${ }^{2}$ School of Nursing and Midwifery, Griffith University, Nathan, QLD, Australia

Full list of author information is available at the end of the article
}

(c) The Author(s). 2018 Open Access This article is distributed under the terms of the Creative Commons Attribution 4.0 International License (http://creativecommons.org/licenses/by/4.0/), which permits unrestricted use, distribution, and reproduction in any medium, provided you give appropriate credit to the original author(s) and the source, provide a link to the Creative Commons license, and indicate if changes were made. The Creative Commons Public Domain Dedication waiver (http://creativecommons.org/publicdomain/zero/1.0/) applies to the data made available in this article, unless otherwise stated. 


\section{Background}

Peripheral intravenous catheter (PVC) insertion is the most commonly performed invasive procedure in hospitalised patients. Worldwide, it is estimated that 2 billion PVCs are sold each year [1] and used for the short-term delivery of intravenous (IV) medications and fluids [2]. However, multiple insertion attempts are common, and post-insertion failures from complications such as occlusion are as high as $69 \%$, triggering the insertion of subsequent catheters [3-5].

PVC inserter models vary. Traditionally, intravenous therapy teams (IVTTs) were used for most PVC insertions [6, 7]. These teams were made up of nurses with advanced skills in insertion and maintenance $[7,8]$. More recently, many IVTTs have been discontinued due to health care budget cuts, leaving PVC insertion to generalist nursing and medical staff [9]. Data supporting cost savings associated with disbanding IVTTs are yet to be reported in literature [9].

The generalist model involves nurses and medical staff at the clinical unit level [8], which ideally enables continuity of care if they are aware of the patient's diagnosis and clinical history. A belief exists that even though generalists may have minimal PVC insertion skills, this rarely leads to negative outcomes [10]. This belief likely stems from a focus on PVC insertion success alone, but there is an ongoing concern that the varying skill level of generalist inserters leads to multiple needle sticks, patient discomfort and irreversible damage to the venous system, limiting current and future vascular access options $[9,11]$.

Other workforce models for PVC insertion include the use of vascular access specialists (VASs), who are practitioners with advanced assessment as well as technical skills for all vascular access devices. These practitioners may work within an IVTT [8] or within a specific unit or nursing framework [12] for device insertion, surveillance, research and education.

For the purpose of this trial, a VAS was defined as a clinician with advanced knowledge of vascular access, including catheter technology (materials and design); insertion assistive devices (such as ultrasonography); dressings; processes of catheter access; and management of IV therapy. This advanced level of expertise and knowledge is believed to preserve veins, enhance the patient experience, decrease the incidence of infusion complications and ultimately save on costs associated with clinician time, PVC-related products and length of hospital stay [12].

Effective PVC placement and the prevention of PVC-related complications are not only important clinical objectives but also essential for patient satisfaction [6]. A recent international survey exploring patients' perspectives on PVC insertion found that consumers wanted standards implemented for inserters in order to feel safe and trust their health care professionals [13]. The level of skill of the PVC inserter is a risk factor for catheter failure $[6,14]$. However, limited high-quality research exists exploring the efficacy of a VAS. Observational studies and audits report that VAS-inserted PVCs have fewer first-time insertion attempts $[6,15]$, less phlebitis [16, 17], less inflammation and catheter-related sepsis [18] and higher patient satisfaction [19]. As some of these studies were conducted before the year $2000[16,17,20]$, it is unclear what impact more advanced PVC materials, dressings and other IV supportive equipment have had on PVC failure. Other observational studies have reported on the success of PVC insertion but not the subsequent catheter failure rate [6], or on the benefit of a VAS within a hospital but not compared to a generalist inserter $[6,7]$. Limitations of previous research include data collected retrospectively [7], secondary analysis of existing datasets [14] or clinical staff assigning their own level of insertion skill [12].

There is a paucity of evidence from high-quality randomised control trials (RCTs) assessing inserter skill levels required for successful PVC insertion and prevention of device failure and complications. This makes it impossible for local and international guideline writers to produce comprehensive clinical practice guidelines for the best PVC insertion model of care. Therefore, it is important to examine the efficacy of different models for PVC insertion used in hospitals.

\section{The study}

We compared standard care (generalist model: PVCs inserted in line with hospital policy by an accredited PVC inserter) with insertion by a VAS. The VAS for this pilot trial was a member of an intravenous therapy team for more than 20 years and an educator training clinicians to place PVCs in both a hospital and university program. The aim of this trial was to test the feasibility of conducting a suitably powered RCT by assessing both the methodology and rigour of methods planned for the larger study.

\section{Methods \\ Study design and participants}

We undertook a single-centre, parallel-group, pilot RCT in a large government teaching hospital in Queensland, Australia. Human research ethics committee approval was obtained from the hospital ethics committee (HREC/16/QRBW/386) and Griffith University (2016/782). The trial was registered with the Australian New Zealand Clinical Trials Registry (ACTRN12616001675415), and the protocol was published [21]. 
We recruited patients admitted to general medical and surgical wards between July and November, 2017. A research nurse $(\mathrm{ReN})$ screened for patients who were over the age of 18, expected to have a PVC for greater than $24 \mathrm{~h}$ and able to provide written and informed consent. We excluded patients who had a current bloodstream infection or who had previously been enrolled in the study.

\section{Sample size}

The recruitment target for this pilot RCT was 69 participants per group. This trial was not designed to have adequate power to detect statistical significances between groups, but rather to assess the feasibility of the methods to be used in a larger study. The sample size is considered appropriate for the purposes of feasibility assessment [22, 23].

\section{Randomisation and masking}

The ReN obtained written informed consent and then, using a web-based central randomisation service (Griffith University Clinical Trials Randomisation Service, www151.griffith.edu.au), obtained group allocation, which was $1: 1$ with randomly varied small block sizes. Allocation was concealed prior to randomisation.

Two ReNs collected data for this trial. The first was responsible for recruitment and randomisation. The second ReN was masked to the study intervention and responsible for the daily PVC site inspections and device failure information. The endpoint of catheter-related bloodstream infection (CRBSI) was assessed by an infectious diseases physician who, along with the study statistician, was masked to group allocation. However, due to the nature of the study, blinding of patients and treating clinicians to the intervention received was not possible.

\section{PVC care and maintenance}

All PVCs were inserted by hospital-accredited clinicians using local hospital policies. Skin decontamination for all insertions was with a 3M (St Paul, MN, USA) SoluPrep $^{\text {Th }}$ Antiseptic Swab (2\% chlorhexidine gluconate [CHG] in 70\% isopropyl alcohol [IPA]). All PVCs were Becton Dickinson (BD; Sandy, UT, USA) Insyte ${ }^{\mathrm{mm}}$ Autoguard $^{\mathrm{Tw}}$ Blood Control (non-winged) catheters with a Smart-Site ${ }^{\mathrm{Tx}}$ Needle-Free Valve (BD) and a 10-cm extension tubing with a bonded three-way connector (Connecta $\left.{ }^{\mathrm{ma}}, \mathrm{BD}\right)$. As per hospital policy, PVCs were to be re-sited every $72 \mathrm{~h}$, unless the clinician chose to extend the dwell time in response to a clinical indication.

\section{Outcome measures}

The primary outcome was to establish the feasibility of an adequately powered RCT using the following criteria:
$>90 \%$ of patients screened would be eligible; $>90 \%$ of eligible patients would agree to enrol; $>90 \%$ of eligible patients would receive the allocated intervention; $<5 \%$ of enrolled patients would be lost to follow-up; and $<5 \%$ missing data.

The secondary outcomes included the following: (1) PVC failure, i.e. catheter removal before the end of therapy due to phlebitis (defined as two or more occurrences of pain, erythema, swelling, palpable cord or purulent discharge), infiltration (movement of IV fluids into the surrounding tissue), occlusion (PVC will not flush or leaks when flushed), accidental removal (partial or complete dislodgement of the PVC from the vein), infection (laboratory-confirmed local or PVC-related bloodstream infection [24]), PVC positive skin swab and/or positive PVC tip culture [25] (as per usual clinical practice); (2) PVC dwell time (from insertion until removal from either PVC failure, routine replacement or the completion of IV therapy); (3) insertion success; (4) insertion attempts; and (5) cost-effectiveness (a sub-set of PVC insertions observed and timed to establish estimates of staff costs and equipment).

\section{Data collection}

Data for this study were collected by a ReN and entered into an electronic data platform supported by REDCap ${ }^{\text {tw }}$ (Research Electronic Data Capture 6.10.6 () 2016 Vanderbilt University, Nashville, TN, USA) [26]. Feasibility outcomes (eligibility, recruitment, retention and attrition, protocol adherence and sample size estimates) were collected from enrolment screening logs.

At participant recruitment the $\mathrm{ReN}$, who was also a VAS, collected patient demographic and clinical characteristics such as age, gender and vein quality as per the peripheral vein assessment tool [27]. From this assessment, taking into consideration the planned IV treatment and patient preference, the ReN/VAS documented their recommendation for vascular access device (VAD) choice and site selection. Participants were then randomised $u$. After the PVC was inserted, the $\mathrm{ReN}$ documented the gauge, profession of inserting clinician, number of insertion attempts, place of insertion and type of securement/dressing applied.

Trial participants were visited daily by the second ReN, who was masked to the intervention group. The ReN assessed patient satisfaction with the insertion procedure on an 11 -point scale $(0=$ not satisfied and $10=$ satisfied $)$. The ReN also inspected the PVC site for redness, swelling and palpable cord (measured in centimetres from insertion site); patient-reported pain/tenderness $(0=$ no pain and $10=$ maximum pain); leakage (yes/no); and purulence (none, from site, with ulceration). At PVC removal, the ReN recorded the date and time and the reason for removal. The participant was then asked to rate their overall 
satisfaction with the catheter on an 11-point scale $(0=$ not satisfied and $10=$ satisfied).

\section{Statistical analysis}

Feasibility outcomes were reported descriptively and analysed against predetermined acceptability criteria. Statistical analysis was performed using Stata 15 (Stata Corp, College Station, TX, USA). An intention-to-treat analysis framework was used; the unit of analysis was one PVC per patient. Missing data were not imputed. Frequencies and proportions were reported for categorical data. Mean values and standard deviations (SDs) were reported for normally distributed data; median values and 25 th/75th percentiles reported otherwise. Covariates were re-categorised to suit the regression analyses as necessary and were not analysed in regression models if they had fewer than 20 cases. A graph of the Kaplan-Meier survival function was generated, and a log-rank test performed. Univariable and multivariable Cox regression was used to assess the effect of patient and treatment differences as well as for group comparisons. Covariates were deemed eligible for multivariable analysis at $p<0.20$ and were dropped from the multivariable model during manual backward model building at $p \geq 0.05$. The proportional hazards assumption was checked. $p$ values $<0.05$ were considered significant.

\section{Results}

\section{Primary outcome}

Between July and November 2017, 150 patients were screened, and $92 \%$ were eligible for trial recruitment. Willingness for study involvement was high, with only two patients declining trial participation. No patients were lost to follow-up, none received the incorrect study allocation and there were no missing outcome events; therefore, all predetermined feasibility criteria (Fig. 1) were met as per the trial protocol [21].

\section{Patient and PVC characteristics}

At recruitment, patients had similar demographic characteristics between groups (Table 1). They were predominantly

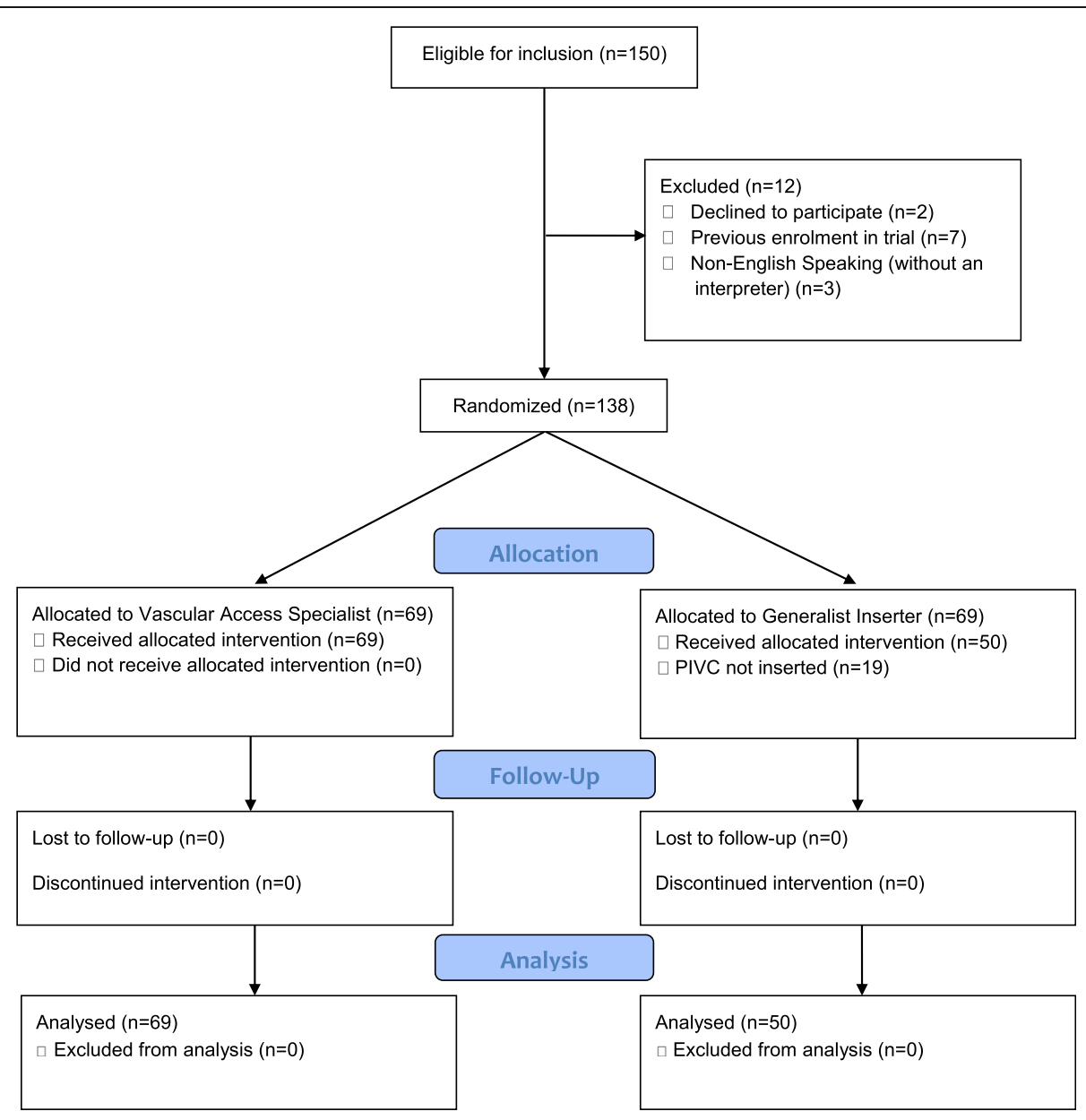

Fig. 1 Consolidated Standards of Reporting Trials (CONSORT) flow chart 
Table 1 Descriptive statistics by study groups

\begin{tabular}{|c|c|c|c|c|c|}
\hline & $n$ & VAS & $n$ & Generalist & Total \\
\hline Group size $^{a}$ & & $69(50)$ & & $69(50)$ & $138(100)$ \\
\hline Age (years) ${ }^{b}$ & 69 & $64.0(47.0-73.0)$ & 69 & $62.0(47.0-71.0)$ & $62.0(47.0-73.0)$ \\
\hline Sex: males & 69 & $43(62)$ & 69 & $43(62)$ & $86(62)$ \\
\hline Weight category: overweight/obese & 69 & $37(54)$ & 69 & $33(48)$ & $70(51)$ \\
\hline Skin integrity: good & 69 & $41(59)$ & 69 & $36(52)$ & $77(56)$ \\
\hline Mobility at insertion: & 69 & & 69 & & \\
\hline Independent & & $35(51)$ & & $53(77)$ & $88(64)$ \\
\hline Required assistance to mobilise & & $21(30)$ & & $9(13)$ & $30(22)$ \\
\hline Bed-bound & & $13(19)$ & & $7(10)$ & $20(14)$ \\
\hline Reason for admission: & 69 & & 69 & & \\
\hline Medical & & $19(28)$ & & $19(28)$ & $38(28)$ \\
\hline Surgical emergent & & $14(20)$ & & $21(30)$ & $35(25)$ \\
\hline Surgical elective & & $36(52)$ & & $29(42)$ & $65(47)$ \\
\hline Infection at recruitment & 69 & $21(30)$ & 69 & $21(30)$ & $42(30)$ \\
\hline Number of comorbidities: & 69 & & 69 & & \\
\hline Zero & & $12(17)$ & & $10(14)$ & $22(16)$ \\
\hline One & & $17(25)$ & & $15(22)$ & $32(23)$ \\
\hline Two & & $11(16)$ & & $17(25)$ & $28(20)$ \\
\hline Three & & $8(12)$ & & $9(13)$ & $17(12)$ \\
\hline Four or more & & $21(30)$ & & $18(26)$ & $39(28)$ \\
\hline Wound (present at recruitment) & 69 & $42(61)$ & 69 & $40(58)$ & $82(59)$ \\
\hline Vein assessment: & 69 & & 69 & & \\
\hline Excellent & & $18(26)$ & & $25(36)$ & $43(31)$ \\
\hline Good & & $14(20)$ & & $19(28)$ & $33(24)$ \\
\hline Fair or poor & & $37(54)$ & & $25(36)$ & $62(45)$ \\
\hline Vein first choice for insertion: & 69 & & 69 & & \\
\hline Cephalic & & $38(55)$ & & $45(65)$ & $83(60)$ \\
\hline Medial antebrachial & & $15(22)$ & & $6(9)$ & $21(15)$ \\
\hline Accessory cephalic & & $8(12)$ & & $8(12)$ & $16(16)$ \\
\hline Other & & $8(12)$ & & $10(14)$ & $18(13)$ \\
\hline Location first choice for insertion: & 69 & & 69 & & \\
\hline Posterior lower forearm & & $32(46)$ & & $45(65)$ & $77(56)$ \\
\hline Upper anterior forearm & & $20(29)$ & & $7(10)$ & $27(20)$ \\
\hline Wrist & & $12(17)$ & & $10(14)$ & $22(16)$ \\
\hline Other & & $5(7)$ & & $7(10)$ & $12(9)$ \\
\hline Device sequence: & 69 & & 69 & & \\
\hline Initial & & $3(4)$ & & $0(0)$ & $3(2)$ \\
\hline Subsequent & & $66(96)$ & & $69(100)$ & $135(98)$ \\
\hline Reason for insertion: & 69 & & 69 & & \\
\hline IV medications only & & $20(29)$ & & $20(29)$ & $40(29)$ \\
\hline IV medications and/or fluids & & $49(71)$ & & $49(71)$ & $98(71)$ \\
\hline PVC is the appropriate device & 69 & $58(84)$ & 69 & $55(80)$ & $113(82)$ \\
\hline Insertion difficulty $(0=\text { none, } 10=\max )^{b}$ & 69 & $2.0(0.0-5.0)$ & 11 & $2.0(1.0-5.0)$ & $2.0(0.5-5.0)$ \\
\hline Pain at insertion $(0=\text { none, } 10=\max )^{b}$ & 69 & $2.0(1.0-3.0)$ & 44 & $3.0(1.0-4.0)$ & $2.0(1.0-3.0)$ \\
\hline
\end{tabular}


Table 1 Descriptive statistics by study groups (Continued)

\begin{tabular}{|c|c|c|c|c|c|}
\hline & $n$ & VAS & $n$ & Generalist & Total \\
\hline Device size (gauge): & 69 & & 50 & & \\
\hline 22 & & $46(67)$ & & $25(50)$ & $71(60)$ \\
\hline 20 & & $21(30)$ & & $19(38)$ & $40(34)$ \\
\hline Other & & $2(3)$ & & $2(4)$ & $4(3)$ \\
\hline Not documented & & $0(0)$ & & $4(8)$ & $4(3)$ \\
\hline Reason for choosing size ${ }^{c}$ : & 69 & & 11 & & \\
\hline Clinician preference & & $41(59)$ & & $10(90)$ & $51(64)$ \\
\hline Patient has limited vein size & & $33(48)$ & & $2(18)$ & $35(44)$ \\
\hline Other & & $13(19)$ & & $1(9)$ & $14(18)$ \\
\hline IV placement: & 69 & & 50 & & \\
\hline Cephalic & & $31(45)$ & & $20(40)$ & $51(43)$ \\
\hline Medial antebrachial & & $16(23)$ & & $3(6)$ & $19(16)$ \\
\hline Accessory cephalic & & $10(14)$ & & $3(6)$ & $13(11)$ \\
\hline Metacarpal & & $3(4)$ & & $10(20)$ & $13(11)$ \\
\hline Other & & $9(13)$ & & $14(28)$ & $23(19)$ \\
\hline IV location: & 69 & & 50 & & \\
\hline Posterior lower forearm & & $26(38)$ & & $13(26)$ & $39(33)$ \\
\hline Upper anterior forearm & & $22(32)$ & & $4(8)$ & $26(22)$ \\
\hline Wrist & & $14(20)$ & & $9(18)$ & $23(19)$ \\
\hline Hand & & $3(4)$ & & $14(28)$ & $17(14)$ \\
\hline Other & & $4(6)$ & & $10(20)$ & $14(12)$ \\
\hline Side of insertion: right & 69 & $38(55)$ & 50 & $23(46)$ & $61(51)$ \\
\hline Skin hair prior to insertion: & 69 & & 50 & & \\
\hline None present & & $31(45)$ & & $31(62)$ & $62(52)$ \\
\hline Clipped & & $38(55)$ & & $3(6)$ & $41(34)$ \\
\hline Unclipped & & $0(0)$ & & $16(32)$ & $16(13)$ \\
\hline
\end{tabular}

Frequencies and column percentages shown, unless otherwise noted

${ }^{\text {a }}$ Row percentages shown

b Median and 25th-75th percentiles shown

c Multiple responses allowed

$n$ number of non-missing observations, VAS vascular access specialist, $I V$ intravenous, PVC peripheral intravenous catheter, max maximum

male, overweight or obese and admitted to a surgical ward. The 22 gauge PVC was more frequently used by VASs (67\%) in comparison to generalists (50\%). Generalists inserted more PVCs into the hand or wrist (46\%) than the forearm (34\%), whereas the VASs placed more catheters into the forearm (70\%) than the hand or wrist (24\%). The generalist inserters were medical staff (82\%), anaesthetists (4\%) and nurses (14\%). The VAS inserter used ultrasound assistance with three insertions. Ultrasound was not used by the generalist group. Multiple insertion attempts occurred more often in the generalist (35\%) than in the VAS group (19\%).

The initial masked vein assessment identified a higher number of participants with fair or poor veins randomised to the VAS insertion group (54\%) compared with $36 \%$ in the generalist group. The ideal site and vein for PVC placement assessed by the VAS prior to randomisation was achieved for $81 \%$ of PVCs placed by the VAS, compared with $26 \%$ of the generalist inserters.

\section{Secondary outcomes}

All PVCs $(n=69)$ were successfully inserted in the VAS group. In the generalist group, 19 (28\%) patients did not have a PVC inserted and, in response, were changed to oral medication $(n=8)$, had a pre-existing PVC left in place $(n=6)$, had a peripherally inserted peripheral catheter inserted $(n=2)$ or remained waiting for a PVC insertion for at least $24 \mathrm{~h}(n=3)$.

PVC insertion timings and procedural resource usage were collected for 16 VAS and 4 generalist insertions. The mean PVC insertion procedure time was $2 \mathrm{~min}$ in the VAS group and $11 \mathrm{~min}$ in the generalist group. A full cost-effectiveness analysis could not be undertaken due 
to limited generalist group data collected as a result of the following: (1) long delays from PVC request to insertion of the catheter; (2) the fact that many patients in the generalist group did not ultimately have a PVC inserted.

PVC post-insertion failure was $54 \%$ in the generalist group and $48 \%$ in the VAS group (Table 2). This equated to 217 and 228 failures per 1000 PIV days respectively (incidence rate ratio 1.05, 95\% confidence interval [CI] 0.61-1.80, Table 2); these results were not different upon

Table 2 Study outcomes $(n=119)$

\begin{tabular}{|c|c|c|c|}
\hline & VAS & Generalist & $p$ value \\
\hline & $n=69$ & $n=50$ & \\
\hline PVC successfully inserted & $69(100)$ & $50(72)$ & \\
\hline Multiple insertion attempts ${ }^{a}$ & $13(19)$ & $16(35)$ & \\
\hline Number of insertion attempts ${ }^{a, b}$ & 1.22 & 1.74 & \\
\hline \multicolumn{4}{|l|}{ Reason for removal: } \\
\hline $\begin{array}{l}\text { Treatment complete without } \\
\text { complication }\end{array}$ & $29(42)$ & $19(38)$ & \\
\hline $\begin{array}{l}\text { Treatment incomplete with } \\
\text { complication }\end{array}$ & $26(38)$ & $22(44)$ & \\
\hline $\begin{array}{l}\text { Treatment completed with } \\
\text { complication }\end{array}$ & $7(10)$ & $5(10)$ & \\
\hline $\begin{array}{l}\text { Routine re-site or theatre } \\
\text { replacement }\end{array}$ & $5(7)$ & $3(6)$ & \\
\hline Insertion of a CVAD & $2(3)$ & $1(2)$ & \\
\hline Device failed & $33(48)$ & $27(54)$ & $0.506^{\mathrm{c}}$ \\
\hline Positive blood count & $0(0)$ & $2(4)$ & \\
\hline \multicolumn{4}{|l|}{ Complication ${ }^{\mathrm{d}}$ : } \\
\hline Phlebitis & $19(28)$ & $10(20)$ & \\
\hline Infiltration & $13(19)$ & $9(18)$ & \\
\hline Occlusion & $7(10)$ & $9(18)$ & \\
\hline Accidental removal & $6(9)$ & $7(14)$ & \\
\hline Unknown & $0(0)$ & $1(2)$ & \\
\hline Device days & 152 & 118 & \\
\hline Incidence rate of failure ${ }^{e, f}$ & $\begin{array}{l}217 \\
(154-305)\end{array}$ & $228(156-332)$ & \\
\hline Incidence rate ratio & Reference & $1.05(0.61-1.80)$ & $0.924^{9}$ \\
\hline \multicolumn{4}{|l|}{ Overall patient satisfaction ${ }^{\mathrm{h}, \mathrm{i}}$} \\
\hline Insertion & $9(8-10)$ & 7 (3.5-9) & \\
\hline Overall & $7(6-9)$ & $4.5(1.5-6)$ & \\
\hline
\end{tabular}

Frequencies and column percentages shown, unless otherwise noted

a Successfully inserted devices only

${ }^{\mathrm{b}}$ Average shown

${ }^{\mathrm{C}} \mathrm{Chi}$-squared test

${ }^{\mathrm{d}}$ Multiple responses allowed

ePer 1000 device days

f Includes 95\% confidence interval

${ }^{g}$ Log-rank test

${ }^{\mathrm{h}}$ Median (25th/75th percentiles) shown

' $0=$ not satisfied, $10=$ satisfied

VAS vascular access specialist, $n$ number of non-missing observations,

$P V C$ peripheral intravenous catheter, CVAD central venous access device
Kaplan-Meier survival analysis (Fig. 2; $\log$-rank $p=0.92$ ). The most common causes of failure were phlebitis and infiltration. Even though this study was not powered to show effect, phlebitis was 5\% higher in VAS-inserted catheters than for generalist insertions. Occlusion and partial or complete dislodgement were higher (absolute $8 \%$ and $5 \%$ respectively) in generalist-inserted PVCs. There were no local or PVC-related bloodstream infections in either group.

Median satisfaction with PVC insertion was higher in the VAS group (9 versus 7) than the generalist group (Table 2). Overall median satisfaction with the PVC was also higher in the VAS group (7 versus 4.5 , Table 2 ) than in the generalist group.

\section{Multivariable modelling for PVC post-insertion failure}

Although this study was not powered to show statistical significances between groups, in the multivariable model (Table 3), medical diagnosis $(p<0.001)$ or bed-bound status at insertion $(p<0.05)$ were associated with an approximately twofold higher incidence of PVC failure, and non-sterile tape securement remained associated with decreased PVC failure (hazard ratio [HR] 0.36, 95\% CI $0.18-0.70, p<0.001)$.

\section{Discussion}

Improving the knowledge and skill of PVC inserters is likely to reduce the current situation where patients commonly experience multiple PVC insertion attempts and unacceptably high post-insertion catheter failure rates. In this pilot RCT, we compared two inserter workforce models for clinical, patient and feasibility outcomes. All predetermined feasibility outcomes were met; thus, we have established that the tested methods are appropriate for an adequately powered, multicentre RCT. Overall PVC failure was higher in the generalist compared with the VAS group, and although pilot trials are not powered for statistical significance, this result was clinically meaningful and needs testing in a larger RCT. To compare $54 \%$ versus $48 \%$ post-insertion failure with $80 \%$ power $(p=0.05)$ would require 1084 patients per group (powerandsamplesize.com).

Under generalist models, establishment of vascular access is frequently left to junior medical and nursing staff who, with minimal knowledge about complications associated with IV medications, may choose a PVC as a default VAD [27, 28]. In our trial, 18\% of PVCs were considered an inappropriate VAD by our blinded VAS assessor, as they had IV therapy prescribed for greater than 5 days and/or poor vascular access. Generalist models also lack standardisation of knowledge and technique for clinicians inserting PVCs, meaning that expertise and maintenance of competence cannot be guaranteed across and within health care settings [29]. 


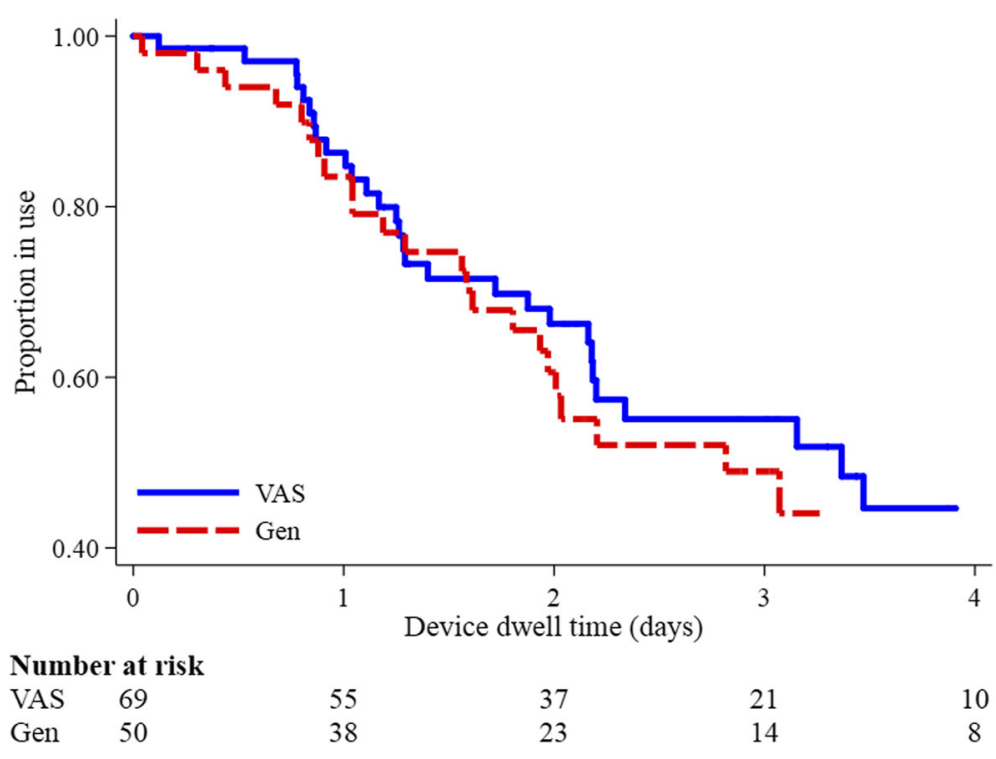

Fig. 2 Kaplan-Meier survival analysis

Such deficiencies likely contributed to the lower first-time PVC insertion success in our generalist group, with multiple insertion attempts occurring almost twice as often with the generalist inserter (35\%) than the VAS inserter (19\%). An additional three patients allocated to the generalist group were still awaiting PVC placement after $24 \mathrm{~h}$ and numerous unpleasant insertion attempts. This is not only a poor patient experience but also has cost implications for clinician time, delayed treatment and potentially extended hospital stay.

More than a quarter of patients allocated to the generalist group in our trial did not receive a PVC, compared to $100 \%$ placement by the VAS group. The purpose of placing a PVC is to start or continue treatment, and this was an unexpected result. It may indicate a lack of PVC insertion skill in the generalist group or a lack of comprehensive assessment regarding the requirement for a PVC. Either way we consider that this may be the more appropriate primary endpoint for a follow-on study. The most commonly reported reason in this study for non-placement was the change of antibiotic therapy from intravenous to oral. The decision to change antibiotic route should be based on three key factors: (1) the antimicrobial agent, (2) the patient and (3) the condition being treated, and careful consideration is necessary to provide the best care [30]. Within this trial it was unclear if these factors were the determining considerations, or if this change was due to the unavailability of staff to place the catheter or unsuccessful insertion attempts rather than the patient's clinical need.

Our multivariable model identified that patients admitted with a medical diagnosis and those who were non-ambulant (bed-bound) were at higher risk of PVC failure. These patients may have a higher risk, as they are likely to have more comorbidities and a history of greater VAD use than ambulant patients and/or those with an acute surgical diagnosis. These patients should be the priority for efforts to improve PVC outcomes. We also found that patients whose PVC was secured with additional non-sterile tape had a significant association with decreased PVC failure. These results reflect a similar finding by a large cohort study at the same hospital that reported significantly lower occlusion/infiltration, phlebitis and dislodgement rates when non-sterile tape was used as an additional PVC securement [31]. This suggests that, despite advances in dressings and securement devices, PVC failure rates have remained high, and even good-quality insertion requires effective securement to maintain function.

\section{Limitations}

The main limitation of this study is that it is a pilot RCT, and although the results are clinically interesting, the study is not designed to provide definitive conclusions about the best model for PVC insertion. The use of the same VAS to perform the pre-randomisation assessment and to insert the PVCs in the VAS group was also a limitation of this study. However, the VAS was blinded to allocation at the time the assessment was conducted, and information was directly entered into an electronic platform at the bedside. 
Table 3 Cox regression

\begin{tabular}{|c|c|c|}
\hline & Univariable & $\begin{array}{l}\text { Multivariable } \\
n=119\end{array}$ \\
\hline Study group: generalist (ref. VAS) & $1.03(0.61-1.73)$ & $1.18(0.70-2.00)$ \\
\hline Sex: male (ref. female) & $0.61(0.35-1.05)^{*}$ & $\#$ \\
\hline Age (increment of 1 year) & $1.00(0.98-1.01)$ & $\wedge$ \\
\hline Body mass index (increment of 1) & $0.99(0.94-1.03)$ & $\wedge$ \\
\hline Comorbidities (none $/ 1 / 2 / 3 / 4$ or more) & $0.96(0.81-1.16)$ & $\wedge$ \\
\hline Insertion on dominant side (ref. no) & $1.03(0.62-1.72)$ & $\wedge$ \\
\hline Bed-bound at insertion (ref. no) & $1.74(0.93-3.24)^{*}$ & $\begin{array}{l}2.17 \\
(1.14-4.11)^{* *}\end{array}$ \\
\hline Medical reason for admission (ref. surgical) & $\begin{array}{l}2.08 \\
(1.22-3.55)^{* * *}\end{array}$ & $\begin{array}{l}2.08 \\
(1.21-3.57)^{* * *}\end{array}$ \\
\hline Infection at recruitment (ref. no) & $0.90(0.52-1.55)$ & $\wedge$ \\
\hline Vein assessment (ref. excellent): & & $\wedge$ \\
\hline Good & $0.70(0.32-1.52)$ & $\wedge$ \\
\hline Fair/poor & $0.34(0.67-2.10)$ & $\wedge$ \\
\hline $\begin{array}{l}\text { Location selected was location in } \\
\text { which PVC was placed (ref. no) }\end{array}$ & $0.79(0.47-1.34)$ & $\wedge$ \\
\hline Difficulty with previous insertion (ref. no) & $0.99(0.56-1.74)$ & $\wedge$ \\
\hline PVC is the appropriate device (ref. no) & $0.72(0.39-1.32)$ & $\wedge$ \\
\hline Device gauge: other (ref. 22) & $0.64(0.36-1.11)^{*}$ & $\#$ \\
\hline Location (ref. posterior lower forearm): & & $\wedge$ \\
\hline Upper anterior forearm & $1.49(0.75-2.97)$ & \\
\hline Wrist & $1.31(0.61-2.79)$ & \\
\hline Hand & $1.89(0.87-4.14)^{*}$ & \\
\hline Other & $0.73(0.27-1.98)$ & \\
\hline Multiple insertion attempts (ref. no) & $0.95(0.53-1.73)$ & $\wedge$ \\
\hline Dressing: non-sterile tape ${ }^{a}$ (ref. never) & $\begin{array}{l}0.38 \\
(0.20-0.74)^{* * *}\end{array}$ & $\begin{array}{l}0.36 \\
(0.18-0.70)^{* * *}\end{array}$ \\
\hline Dressing: Tubigrip ${ }^{a}$ (ref. never) & $0.81(0.46-1.42)$ & $\wedge$ \\
\hline Dressing dirty/wet/damaged ${ }^{\text {a }}$ (ref. never) & $0.89(0.42-1.85)$ & $\wedge$ \\
\hline Fluids ${ }^{a}$ (ref. never) & $1.00(0.59-1.68)$ & $\wedge$ \\
\hline Antibioticsa, b (ref. never) & $1.35(0.72-2.51)$ & $\wedge$ \\
\hline Anaesthesiaa (ref. never) & $0.78(0.42-1.46)$ & $\wedge$ \\
\hline Cefazolin $^{\mathrm{a}}$ (ref. never) & $0.87(0.47-1.62)$ & $\wedge$ \\
\hline Pain relief ${ }^{a}$ (ref. never) & $0.97(0.56-1.66)$ & $\wedge$ \\
\hline $\begin{array}{l}\text { Other IV medication }{ }^{\mathrm{a}, \mathrm{c}} \\
\text { (ref. never) }\end{array}$ & $0.71(0.38-1.31)$ & $\wedge$ \\
\hline $\begin{array}{l}\text { Antiemetic and antireflux }{ }^{a} \\
\text { (ref. never) }\end{array}$ & $0.93(0.50-1.73)$ & $\wedge$ \\
\hline $\begin{array}{l}\text { Accesses (total, none } / 1 \\
\text { to } 3 / 4 \text { to } 6 / 7 \text { or more) }\end{array}$ & $0.85(0.69-1.05)^{*}$ & $\#$ \\
\hline
\end{tabular}

Hazard ratios and $95 \%$ confidence intervals shown

${ }^{*} p$ value $<0.20,{ }^{* *} p$ value $<0.05,{ }^{* * *} p$ value $<0.01$

\# dropped from multivariable model at $p \geq 0.05, \wedge$ ineligible for

multivariable analysis at overall $p \geq 0.20$

${ }^{\text {aAt }}$ any time during study

'Includes ampicillin, benzylpenicillin, gentamicin, vancomycin, ceftazidime, azithromycin, meropenem, cefepime, or augmentin

Includes Frusemide, contrast, insulin, magnesium, or thiamine

VAS vascular access specialist, $P V C$ peripheral intravenous catheter

\section{Conclusion}

This study suggests that less insertion failure and less post-insertion failure occur when catheters are placed by a VAS. This pilot trial has confirmed the feasibility and clinical need for a large, multicentre RCT to test these PVC insertion models to provide evidence for health service delivery improvements.

\section{Abbreviations}

CRBSI: Catheter-related bloodstream infection; HR: Hazard ratio;

IV: Intravenous; IVTT: Intravenous therapy team; PVC: Peripheral intravenous catheter; RCT: Randomised controlled trial; ReN: Research nurse; SD: Standard deviation; VAS: Vascular access specialist

\section{Acknowledgements}

The researchers acknowledge and thank the nurses and patients involved in this study. We would also like to acknowledge and thank BARD Australia for loaning us an ultrasound scanner to assist our VAS.

\section{Funding}

We acknowledge the Royal Brisbane and Women's Hospital and Royal Brisbane and Women's Hospital Foundation for a research project grant to support this trial.

\section{Availability of data and materials}

The datasets generated during the current study are not publicly available due to patient privacy and Human Research Ethics Committee restrictions. Datasets may be available from the corresponding author upon reasonable request.

\section{Authors' contributions}

NM, JW, MC and CMR were involved in study conception and protocol design. NM, JW, EL and CMR were applicants for grant funding. All authors were involved in the interpretation of results and preparation of the manuscript. All authors read and approved the final manuscript.

\section{Ethics approval and consent to participate}

Ethical approval was obtained from Queensland Health (EC00172) (HREC/16/ QRBW/386) and Griffith University (EC00162) (2016/782). Informed consent was provided by all participants.

\section{Consent for publication}

Not applicable.

\section{Competing interests}

NM's employer has received the following on her behalf from manufacturers of peripheral intravenous catheter products: investigator-initiated research grants and unrestricted educational grants from 3M, Adhezion, BD, Centurion Medical Products, Cook Medical, Entrotech and Teleflex.

EL's employer has received the following on her behalf from manufacturers of vascular device products: consultancy payment for an educational lecture from $3 \mathrm{M}$ and an investigator-initiated research grant from Medtronic.

JG's employer has received on her behalf an investigator-initiated research grant from Centurion Medical Products.

MC's employer has received the following on her behalf from manufacturers of peripheral intravenous catheter products: investigator-initiated research grants and unrestricted educational grants from Baxter, BD, Centurion Medical Products and Entrotech.

CMR's employer has received the following on her behalf from manufacturers of peripheral intravenous catheters or vascular device dressing and securement products: investigator-initiated research or educational grants from 3M, Adhezion, AngioDynamics, Bard, Baxter, B Braun, BD, Centurion Medical Products, Cook Medical, Entrotech, FloMedical, ICU Medical, Medical Australia, Medtronic, Smiths Medical and Teleflex; and consultancy payments for educational lectures/expert advice from 3M, Bard, B Braun, BD, ResQDevices and Smiths Medical.

The remaining authors declare that they have no competing interests. 


\section{Publisher's Note}

Springer Nature remains neutral with regard to jurisdictional claims in published maps and institutional affiliations.

\section{Author details}

${ }^{1}$ Royal Brisbane and Women's Hospital, Herston, QLD, Australia. ${ }^{2}$ School of Nursing and Midwifery, Griffith University, Nathan, QLD, Australia. ${ }^{3}$ Alliance for Vascular Access Teaching and Research Group, Menzies Health Institute Queensland, Brisbane, QLD, Australia. ${ }^{4}$ School of Nursing, Queensland University of Technology, Kelvin Grove, QLD, Australia. ${ }^{5}$ School of Medicine, Griffith University, Gold Coast, QLD, Australia. ${ }^{6}$ Centre for Applied Health Economics, Menzies Health Institute Queensland, Brisbane, QLD, Australia.

Received: 14 May 2018 Accepted: 26 September 2018

Published online: 17 October 2018

\section{References}

1. Rickard CM, Ray-Barruel G. Peripheral intravenous catheter assessment: beyond phlebitis. Lancet Haematol. 2017;4(9):e402-3. https://doi.org/10. 1016/S2352-3026(17)30145-X

2. Chopra V, Flanders SA, Saint S, Woller SC, O'Grady NP, Safdar N, et al. The Michigan Appropriateness Guide for Intravenous Catheters (MAGIC): results from a multispecialty panel using the RAND/UCLA appropriateness method. Ann Intern Med. 2015;163(Suppl 6):S1-40. https://doi.org/10.7326/M15-0744.

3. Royer TF. Improving short peripheral IV outcomes: a clinical trial of two securement methods. JAVA. 2003:8(4):45-9. https://doi.org/10.2309/ 155288503774651805.

4. Smith B. Peripheral intravenous catheter dwell times: a comparison of 3 securement methods for implementation of a 96-hour scheduled change protocol. J Infus Nurs. 2006;29(1):14-7.

5. Rickard CM, McCann D, Munnings J, McGrail MR. Routine resite of peripheral intravenous devices every 3 days did not reduce complications compared with clinically indicated resite: a randomised controlled trial. BMC Med. 2010;8:53. https://doi.org/10.1186/1741-7015-8-53.

6. Carr PJ, Glynn RW, Dineen B, Kropmans TJ. A pilot intravenous cannulation team: an Irish perspective. Br J Nurs. 2010;19(10):S19-27.

7. Bosma TL, Jewesson PJ. An infusion program resource nurse consult service: our experience in a major Canadian teaching hospital. J Infus Nurs. 2002; 25(5):310-5.

8. Carr PJ, Higgins NS, Cooke ML, Mihala G, Rickard CM. Vascular access specialist teams for device insertion and prevention of failure. Cochrane Database Syst Rev. 2018;20(3):CD011429. https://doi.org/10.1002/14651858. CD011429.pub2.

9. Hadaway L, Dalton L, Mercanti-Erieg L. Infusion teams in acute care hospitals: call for a business approach: an Infusion Nurses Society white paper. J Infus Nurs. 2013;36(5):356-60. https://doi.org/10.1097/NAN. 0b013e3182a123a9.

10. Robertson KJ. The role of the i.v. specialist in health care reform. J Intraven Nurs. 1995;18(3):130-44.

11. Hawes ML. A proactive approach to combating venous depletion in the hospital setting. J Infus Nurs. 2007;30(1):33-44.

12. Palefski SS, Stoddard GJ. The infusion nurse and patient complication rates of peripheral-short catheters: a prospective evaluation. J Intraven Nurs. 2001; 24(2):113-23.

13. Cooke M, Ullman AJ, Ray-Barruel G, Wallis M, Corley A, Rickard CM. Not "just" an intravenous line: consumer perspectives on peripheral intravenous cannulation (PIVC). An international cross-sectional survey of 25 countries. PLoS One. 2018;13(2):e0193436. https://doi.org/10.1371/journal.pone. 0193436.

14. Wallis MC, McGrail M, Webster J, Marsh N, Gowardman J, Playford EG, et al. Risk factors for peripheral intravenous catheter failure: a multivariate analysis of data from a randomized controlled trial. Infect Control Hosp Epidemiol. 2014;35(1):63-8. https://doi.org/10.1086/674398.

15. Hunter MR. Development of a vascular access team in an acute care setting. J Infus Nurs. 2003;26(2):86-91.

16. Tomford JW, Hershey CO, McLaren CE, Porter DK, Cohen DI. Intravenous therapy team and peripheral venous catheter-associated complications. A prospective controlled study. Arch Intern Med. 1984;144(6):1191-4.

17. Scalley RD, Van CS, Cochran RS. The impact of an i.v. team on the occurrence of intravenous-related phlebitis. A 30-month study. J Intraven Nurs. 1992;15(2):100-9.
18. Soifer NE, Borzak S, Edlin BR, Weinstein RA. Prevention of peripheral venous catheter complications with an intravenous therapy team: a randomized controlled trial. Arch Intern Med. 1998;158(5):473-7.

19. Bai XH, Zang S, Yu L. A comparison of two intravenous infusion devices in lung carcinoma patients receiving combined radiotherapy and chemotherapy. J Cancer Res Ther. 2013;9(4):664-7. https://doi.org/10.4103/ 0973-1482.126475

20. Miller JM, Goetz AM, Squier C, Muder RR. Reduction in nosocomial intravenous device-related bacteremias after institution of an intravenous therapy team. J Intraven Nurs. 1996;19(2):103-6.

21. Marsh N, Webster J, Cooke M, Rickard CM. The RELIABLE trial (RELiable Intravenous Access By Line Experts): a pilot randomised controlled trial protocol of expert versus generalist peripheral intravenous catheter insertion. Vascular Access. 2017;3(2):3-7.

22. Hertzog MA. Considerations in determining sample size for pilot studies. Res Nurs Health. 2008;31(2):180-91. https://doi.org/10.1002/nur.20247.

23. Julious SA. Sample size of 12 per group rule of thumb for a pilot study. Pharm Stat. 2005;4(4):287-91. https://doi.org/10.1002/pst.185.

24. O'Grady NP, Alexander M, Burns LA, Dellinger EP, Garland J, Heard SO, et al. Guidelines for the prevention of intravascular catheter-related infections. Am J Infect Control. 2011;39(4, Suppl 1):S1-34.

25. Maki DG, Weise CE, Sarafin HW. A semiquantitative culture method for identifying intravenous-catheter-related infection. N Engl J Med. 1977; 296(23):1305-9.

26. Harris PA, Taylor R, Thielke R, Payne J, Gonzalez N, Conde JG. Research electronic data capture (REDCap) —a metadata-driven methodology and workflow process for providing translational research informatics support. J Biomed Inform. 2009;42(2):377-81. https://doi.org/10.1016/j.jbi.2008.08.010.

27. Hallam C, Weston V, Denton A, Hill S, Bodenham A, Dunn H, et al. Development of the UK Vessel Health and Preservation (VHP) framework: a multi-organisational collaborative. J Infect Prev. 2016;17(2):65-72. https://doi. org/10.1177/1757177415624752.

28. Jackson T, Hallam C, Corner T, Hill S. Right line, right patient, right time: every choice matters. Br J Nurs. 2013;22(Sup5):S24-8.

29. Vizcarra C, Cassutt C, Corbitt N, Richardson D, Runde D, Stafford K. Recommendations for improving safety practices with short peripheral catheters. J Infus Nurs. 2014:37(2):121-4. https://doi.org/10.1097/NAN. 0000000000000028

30. Beíque L, Zvonar R. Addressing concerns about changing the route of antimicrobial administration from intravenous to oral in adult inpatients. Can J Hosp Pharm. 2015;68(4):318-26.

31. Marsh N, Webster J, Larsen E, Cooke M, Mihala G, Rickard CM. Observational study of peripheral intravenous catheter outcomes in adult hospitalized patients: a multivariable analysis of peripheral intravenous catheter failure. J Hosp Med. 2017:E1-7. https://doi.org/10.12788/jhm.2867.

Ready to submit your research? Choose BMC and benefit from:

- fast, convenient online submission

- thorough peer review by experienced researchers in your field

- rapid publication on acceptance

- support for research data, including large and complex data types

- gold Open Access which fosters wider collaboration and increased citations

- maximum visibility for your research: over $100 \mathrm{M}$ website views per year

At $\mathrm{BMC}$, research is always in progress.

Learn more biomedcentral.com/submissions 